\title{
In Case of Investigator Burnout...
}

Running fast and getting nowhere. That was how Brenda McNulty described her research. She knew what she had to do to get the answer to her questions on cancer angiogenesis, and she worked hard, but experiment after experiment was a failure. McNulty was an experienced investigator, and she could not find any logical explanation for her problems. She reviewed and re-reviewed her methodology, her technicians' procedures, cell lines and their quality, the type of animals she used, the health of the animals and their husbandry conditions, all to no avail. Consultations with colleagues at other institutions were of little help, because they felt that she was doing nothing wrong. They used the same type and source of transgenic animals McNulty used, but they were not having problems with their work. Nevertheless, she simply could not get usable data. After a while, she came to the conclusion that the best course of action would be to take a sabbatical and start anew a few months later. Without any further discussion with the Great Eastern University animal care facility personnel, she depopulated her entire breeding colony of more than 200 mice, and left to work in a colleague's laboratory.

Needless to say, the animal facility personnel felt more than a little bitterness. At the least, they would have wanted to try to transfer many of the animals to another study, but that opportunity was no longer available. It was true that McNulty had not appeared to have done anything that violated her IACUC protocol, but everybody felt she could have worked with them to make better use of the mice.

Six months later McNulty returned, feeling invigorated and ready for a fresh start. Each of her studies required no less than 25 of the transgenic mice, so she placed an order for two breeding pairs of mice from a colleague, and prepared to restart her colony. When the supervisor of animal care became aware of the order, she immediately complained to the animal facility director that this would just lead to more dead animals. The director did not want to be judgmental, so he brought the concern to the Chairman of the IACUC. The IACUC Chairman did not want full responsibility for the decision-making, so he brought the concern to the full Committee. The Committee asked McNulty if she was planning to do anything different from what she had done before she left. She said no, but she had been overly stressed before she left, and now, being more relaxed, she was confident that her studies would succeed. The supervisor of animal care, who was a member of the IACUC, reminded the Committee that this research involved more than 25 mice. To acquire 25 usable study animals, McNulty would have to breed many more to get those having the correct genotype, and even more than that, because she only used females.

McNulty had an approved protocol and a lot of confidence. Recent problems aside, her animal work had been productive, but now she was simply resuming her pre-sabbatical efforts. Is there any reason for the IACUC to revisit its approval or even rescind it? How should the IACUC handle this situation?

\section{Reveal the Game Plan}

\section{Larry Carbone, DVM, PhD, LAT}

The first issue is relatively simple, in that the IACUC should certainly revisit decisions it has made during the life of an approved protocol any time there are serious concerns about animal welfare. An approval letter from the IACUC in no way grants special immunity from ongoing oversight as new circumstances arise. Those circumstances can include unexpected adverse effects on animal health and welfare, new guidelines for animal care and use, and other unforeseen issues. It is highly unlikely that McNulty's protocol included elimination and re-establishment of a breeding colony as originally approved.

The IACUC should assess McNulty's course of action in light of two issues: (1) the extent to which her animals might have been in demand at the time she chose to eliminate the colony, and (2) the likelihood that she thought she would resume this line of work upon her return to campus.

If there had been high demand for her animals, she should have transferred them, if not within her own institution, then to colleagues on other campuses. However, her animals may well have been a specialized line of little use to others, and another institution (such as the one from which she is getting new foundation animals) may not have been able or willing to take them.

McNulty may have honestly believed that it was improbable she would return to this line of investigation. If so, there are welfare costs to maintaining a colony "on speculation" just as surely as there are costs to depopulating and repopulating. To maintain the colony might have required ongoing breeding, selection of new young replacement stock, and euthanasia of unneeded litters of animals. These costs escalate if she has no technical support staff on campus to maintain the colony and is not herself present to do the work. If she truly thought that she would not be returning to this line of work, depopula- 